REVIEW

\title{
Endocrine emergencies
}

\author{
M W Savage, P M Mah, A P Weetman, J Newell-Price
}

Postgrad Med J 2004;80:506-515. doi: 10.1136/pgmj.2003.013474

Diabetic and endocrine emergencies are traditionally treated by the acute medical admitting team or accident and emergency department staff. Most will see diabetic emergencies on a regular basis, as they are common and both type 1 and type 2 disease are increasing in prevalence. Diabetic emergencies are usually easily treated and the patients discharged. However, it is vital not to become complacent as these disorders can lead to death. It is particularly important to follow local guidance and to involve the diabetes team both during and after each episode. Recently it has become clear that about $30 \%$ of patients admitted with acute coronary syndrome (including infarction) have either diabetes or "stress

hyperglycaemia"; evidence suggests that these patients should be treated not only as a cardiac emergency but also as a diabetic one. Thus, every patient with acute coronary syndrome or acute myocardial infarction needs diabetes to be excluded. The other endocrine emergencies are less common, but in some ways more important simply because of their rarity. A high level of suspicion is often required to make a diagnosis, although some, such as myxoedema coma, are usually obvious. Treatment must be started before the diagnosis can be confirmed. Guidance on making the diagnosis and initiating treatment should be made available on the local NHS intranet for nonendocrinologists to access; and where possible expert advice made available by telephone. The basic management steps in the common diabetic and endocrine emergencies are outlined; this is not a complete list, but rather an insight for those involved in non-selected emergency admissions.

See end of article for authors' affiliations

Correspondence to: Dr Mark W Savage, Diabetes Centre, North Manchester General Hospital, Delauneys Road, Manchester M8 5RB, UK; mark.savage@pat.nhs.uk

Submitted

20 November 2003

Accepted

21 February 2004
$\mathrm{D}$ iabetic emergencies are common while non-diabetic endocrine emergencies are rarer and require a high index of suspicion for diagnosis and management especially when presenting to emergency departments. Treatment of the latter must be started before diagnostic confirmation. There is limited evidence based assessment of management in the literature and here we outline management guidelines used in clinical practice for the following conditions:

Diabetes: diabetic ketoacidosis; hyperosmolar non-ketotic "coma" "HONK" (also known as hyperglycaemic hyperosmolar state); hypoglycaemia; and hyperglycaemia in acute coronary syndromes/myocardial infarction.
Endocrinology: adrenal (acute adrenocortical insufficiency, phaeochromocytoma crisis); hypercalcaemia; thyroid (myxoedema coma, thyroid storm); and pituitary (pituitary apoplexy).

\section{DIABETES}

\section{Diabetic ketoacidosis (DKA)}

This is a condition characterised by hyperglycaemia, ketosis, and acidosis. Hyperglycaemia is a result of severe insulin deficiency, either absolute or relative, which impairs peripheral glucose uptake and promotes fat breakdown; relative glucagon excess promotes hepatic gluconeogenesis. Ketosis is caused by a switch to fat metabolism, leading to free fatty acid oxidation in the liver forming the ketone bodies acetoacetic acid and 3-hydroxybutyric acid. The dissociation of the ketone bodies (weak acids) results in acidosis. DKA may occur in patients with known type 1 diabetes, or be the presenting complex leading to the diagnosis.

\section{Clinical features}

The clinical symptoms and signs are predictable from an understanding of the pathophysiology. Hyperglycaemia leads to thirst and polyuria with variable circulatory stress, ranging from normal pulse rate and blood pressure through to severe collapse. Other factors, such as sepsis, may also impact on the degree of shock. Ketosis leads to a smell of acetone on the breath. Acidosis, with an increased anion gap, leads to compensatory respiratory alkalosis with tachypnoea. Precipitating causes increase the circulating levels of stress hormones such as epinephrine, growth hormone and cortisol, and the resulting increase in insulin resistance reduces the effectiveness of any residual insulin production or injected insulin. Examples of precipitants are: pneumonia; myocardial infarction; urinary tract infections; influenza; surgical emergencies; etc. These conditions often present with their own complex of symptoms and signs. However, it must not be forgotten that errors of insulin administration are often the cause, and indeed, patients' manipulation of their insulin treatment is not uncommon. ${ }^{1}$

\section{Diagnosis}

Patients usually present with DKA in the context of a known diagnosis of type 1 diabetes. More

Abbreviations: $\mathrm{ACTH}$, adrenocorticotrophic hormone; DKA, diabetic ketoacidosis; ECG, electrocardiogram; HDU, high dependency unit; HONK, hyperosmolar non-ketotic coma; PAl, primary adrenal insufficiency; PTH, parathyroid hormone; SAl, secondary adrenal insufficiency 


\section{Box 1: Catches for the unwary in DKA}

- Ketones may be apparently absent in urine during the early stages of DKA due to the "Ketostix" inability to detect 3-hydroxybutyric acid, which may initially be the main ketone body in the urine. If in doubt plasma ketones should be measured. The laboratory based tests usually only detect acetoacetone but with higher sensitivity than urine testing.

- If there is any suggestion that DKA is not the reason for the acidosis, serum salicylate and lactate should be measured.

- Blood glucose may not be very high due to partial treatment with insulin before admission, either by the patient or primary care physician.

- Not everyone can smell acetone to the same degree, assess yours by comparison with colleagues' ability to smell commercial acetone-for example, nail varnish remover.

- Pseudohyponatraemia may result from highly lipaemic serum - if the serum sodium is low this should be checked with the laboratory.

- Serum potassium levels may be initially very high as acidosis drives potassium into the extracellular compartment and levels will fall (sometimes rapidly) as treatment continues and acidosis resolves.

rarely these days, at least in the developed world, they may be a newly presenting case. Diagnosis is confirmed by three features. (1) There is a raised random blood glucose concentration greater than $11.1 \mathrm{mmol} / \mathrm{l}$. It is usually, but not always, greater than $15 \mathrm{mmol} / \mathrm{l}$ and is generally below $44 \mathrm{mmol} / \mathrm{l}$. (2) The presence of ketonaemia can be inferred by the presence of large quantities of ketones in the urine checked with "Ketostix". It is important to note that significant ketonaemia leads to large amounts of ketones in the urine, and a "trace" on the stick is not sufficient evidence of ketonaemia. (3) The presence of acidosis is best confirmed by estimation of venous bicarbonate; a level below $15 \mathrm{mmol} / \mathrm{l}$ confirms acidosis.

Modern blood glucose meters are now able to measure 3hydroxybutyric acid allowing earlier confirmation of ketonaemia. ${ }^{2}$ The use of these meters, while not yet common, is increasing. Venous bicarbonate can be measured quickly by running a heparinised venous blood sample through a blood gas machine; some of these machines can now also give estimates of electrolytes. Arterial blood gases are not required unless there is a reduced level of consciousness or respiratory distress/disease suspected. Box 1 shows some catches for the unwary.

\section{Treatment}

This revolves around intravenous fluids and low dose insulin infusion. ${ }^{3}$

It is important for the future management of patients not to label them as having DKA unless they truly do, as intensive and intrusive over-management of hyperglycaemia alone, in the absence of ketosis or acidosis, is inappropriate.

\section{Intravenous fluids and insulin infusion}

There are many regimens in many standard textbooks, as well as in the clinically orientated handbooks carried by many junior doctors. There is little, or nothing, to choose between the various "sliding scales" in the public domain. (see box 2 for an example of such a regimen used in our hospitals). Intravenous fluids should start with $0.9 \%$ saline solution unless there is clinical shock, in which case intravenous colloids should be considered. The saline infusion should initially be relatively rapid, about 1 litre per hour for the first two hours, thereafter it can usually be slowed. Most physicians recommend erring on the side of slight under-replacement of the fluid deficit in the first 24 hours, particularly in the young adult or adolescent who is at greater risk of cerebral oedema. It has, however, not been proven that over-enthusiastic fluid replacement is responsible for the occasional case of cerebral oedema, and risk factors for this include: high initial urea concentrations, low carbon dioxide pressure during artificial ventilation, and treatment with bicarbonate. $^{4}$

If the patient is confused, hypoxic or hypotensive, then management in the high dependency unit (HDU) is mandatory.

Blood glucose should be monitored hourly using capillary readings from an approved meter and ensuring the blood sample is taken properly. When capillary glucose readings fall to $<15 \mathrm{mmol} / \mathrm{l}$ normal saline infusions should be replaced with $5 \%$ dextrose.

\section{Bicarbonate}

Among diabetologists, it is now the majority view that intravenous bicarbonate should usually be avoided; some even suggest that if blood gases are not measured the $\mathrm{pH}$ will

\section{Box 2: Fluids and insulin in treatment of DKA and} HONK

Intravenous fluids:

- Give 1 litre $0.9 \%$ saline solution during the first hour.

- If hypotension, give a plasma expander.

- Rate of fluids thereafter depends on age/fitness of patient, typically:

- 1 litre in next 2 hours

- 1 litre in next 4 hours

- then 1 litre 6 hourly

- Reduce rate in elderly/cardiac disease/mild DKA (bicarbonate >10).

- More rapid infusion increases risk of respiratory distress syndrome.

- Switch to $5 \%$ dextrose 1 litre 8 hourly once blood glucose $\leqslant 15 \mathrm{mmol} / \mathrm{l}$; continue $0.9 \%$ saline if still volume deplete.

- If serum sodium rises above $155 \mathrm{mmol} / \mathrm{l}$ switch to $0.45 \%$ saline, or dextrose $5 \%$ if blood glucose $\leqslant 15$ $\mathrm{mmol} / \mathrm{l}$.

Insulin:

- Dilute 50 units of soluble insulin to $50 \mathrm{ml} 0.9 \%$ saline in a syringe.

- Infuse intravenously, using syringe driver, at start rate 6 units/hour; give 10 units intramuscularly if delay in starting intravenous infusion.

- Check venous glucose at 2 hour; if glucose has not fallen check pump working and iv connections, then increase to $12 \mathrm{U} /$ hour.

- Measure blood glucose hourly.

- Once glucose falling, adjust insulin infusion rate according to sliding scale (see table 1). 


\section{Box 3: Use of bicarbonate in DKA}

Potential harmful effects:

- Possible hypokalaemia and arrhythmia.

- Exacerbation of intracellular and intracerebral acidosis due to carbon dioxide's ability to cross cell membranes and blood-brain barrier, when bicarbonate cannot.

Potential benefits:

- Reduced cardiac irritability.

- Reduced respiratory discomfort.

- Reduction in intravenous chloride load.

not be known and thus it is less likely to be given. There are theoretical reasons for not giving this to treat acidosis (see box 3) and one controlled trial that suggests there is no advantage in those with moderately severe acidosis. ${ }^{5}$ No reliable data exist in those whose $\mathrm{pH}$ is less than 6.9.

If it is to be given, it is recommended that $8.4 \%$ solution is infused into a central vein, or give $1.26 \%$ solution in a larger volume peripherally. The amount of bicarbonate that is given should just be enough to raise the $\mathrm{pH}$ to a maximum of 7.2 as alkalosis may result as ketone bodies are metabolised.

It is also recommended that $20 \mathrm{mmol}$ of potassium be given for every $100 \mathrm{mmol}$ of bicarbonate. The issues are highlighted in box 3 .

\section{Moving to subcutaneous insulin}

Intravenous insulin and fluids should be continued until the bicarbonate level is normal, the patient is eating and drinking, and for at least 24 hours. It is not necessary to wait for the urine to become clear of ketones as this usually takes longer. The exact regimen of subcutaneous insulin to be used depends on whether it is a new presentation or a known diabetic patient and should be adjusted as required after expert diabetology assessment.

\section{Further assessment}

Identification of the precipitating cause is essential in order to attempt to prevent recurrence of this life threatening complication. Notably, direct inquiry about insulin omission should be made as many patients stop their insulin if they are not eating; this is the wrong thing to do and all educated and empowered patients will have been told of this. Nevertheless, it is not uncommon. Early assessment by a diabetes specialist nurse and, if required, a dietitian is mandatory. Diabetes education can prevent further episodes of DKA by ensuring that the patient knows appropriate "sick day rules" and how to adjust insulin when unwell. Advice on the use of "Ketostix", or a modern blood glucose/ketone measuring device, can also be given as part of this. Something as simple as a contact telephone number may be all that is required. It is likely that detailed education of type 1 patients with

Table 1 Example of insulin sliding scale prescription

\begin{tabular}{ll}
\hline Blood glucose (mmol/l) & Insulin dose (units/hour) \\
\hline$>17$ & 6 \\
$>11-16.9$ & 4 \\
$>9-10.9$ & 3 \\
$>7-8.9$ & 2 \\
$>4-6.9$ & 1 \\
$<4$ & 0 (or 0.5$)$ \\
\hline
\end{tabular}

Table 2 Guide to potassium replacement

\begin{tabular}{ll}
\hline $\begin{array}{l}\text { Serum potassium } \\
\text { (mmol/l) }\end{array}$ & $\begin{array}{l}\text { Potassium chloride/I intravenous } \\
\text { fluids }\end{array}$ \\
\hline$>5.5$ & Nil and check potassium in 2 hours \\
$4-5.5$ & $20 \mathrm{mmol}$ \\
$<4$ & $40 \mathrm{mmol}$ \\
\hline
\end{tabular}

education tools, such as Dose Adjustment For Normal Eating (DAFNE), may lead to reduced patient errors and so a lower prevalence of DKA. There is presently no evidence for this in a randomised controlled trial, but it is the authors' belief that it would be surprising if this were not the case.

\section{Potassium}

Almost all patients with DKA will be potassium depleted and it is recommended that potassium replacement be given at a standard dose of $20 \mathrm{mmol} / \mathrm{l}$ per litre of saline/dextrose as soon as the first series of biochemical results are known. As with the insulin sliding scales, there are many slight variations on how to replace potassium. It is, however, most important to measure it regularly, two hourly initially, and to adjust the rate of replacement appropriately (see table 2 ). Use of a volume and rate measuring device is mandatory when giving potassium (if available).

\section{HONK or hyperglycaemic hyperosmolar state}

This condition is characterised by hyperglycaemia and high plasma osmolalility with ketoacidosis being absent or less marked. This most commonly occurs in type 2 diabetes and is typically of gradual onset. This is a partial misnomer as patients presenting with this are rarely in a coma due to diabetes. There is usually an underlying medical condition that has exacerbated the (often undiagnosed) type 2 diabetes. The blood glucose rises slowly, but inexorably, over a number of days and the patient becomes progressively more unwell. In most cases there is enough circulating insulin to prevent ketone formation, and therefore there is no acidosis due to ketonaemia; however, if they are very ill lactic acidosis may supervene. The serum osmolality is very high, usually greater than $340 \mathrm{mosm} / \mathrm{kg}$ with a blood glucose often in excess of $50 \mathrm{mmol} / \mathrm{l}$.

Serum osmolality is estimated as $2([\mathrm{Na}]+[\mathrm{K}])+$ [Glucose]. Some authorities also add the serum urea, but this contributes little to tonicity in vivo and may be omitted. Serum osmolality can be measured by the laboratory and if the difference between measured osmolality and estimated osmolality is significant, that is, $>20 \mathrm{mosm} / \mathrm{kg}$, it suggests the presence of another solute, for example alcohol, which can be a precipitant for both DKA and HONK.

\section{Box 4: Treatment of hypoglycaemia}

If conscious:

- $200 \mathrm{ml}$ oral "Lucozade" / $200 \mathrm{ml}$ carton of fruit juice/ 5 teaspoons of sucrose in water/ 6 dextrose tablets.

- "Hypostop".

Reduced conscious level and unable to take oral treatment:

- Intramuscular glucagon $1 \mathrm{mg}$.

- Intravenous $50 \%$ glucose, $50 \mathrm{ml}$ into a large bore cannula. 


\section{Box 5: Notes on hypoglycaemia}

- Home meters often not as accurate below $4 \mathrm{mmol} / \mathrm{l}$.

- Glucagon contraindicated in sulphonylurea induced hypoglycaemia.

- If no recovery in 20 minutes reconsider diagnosis and consider further intravenous glucose.

- Consider admission if secondary to sulphonylurea.

Traditionally the vast majority of patients with HONK are elderly, but there is an awareness that this may occasionally occur in children as the prevalence of type 2 diabetes is rising rapidly in this group.

Treatment is essentially the same as for DKA, but with some modifications, in particular one can use $0.45 \%$ saline as the initial replacement fluid if the serum sodium is greater than $155 \mathrm{mmol} / \mathrm{l}$. Insulin replacement is required initially at a maximum of $6 \mathrm{U} /$ hour, but with volume replacement the blood glucose levels fall rapidly and after recovery not all patients require insulin treatment.

Mortality was $17 \%$ in one study, but is associated with preexisting comorbidity. ${ }^{6}$ While thromboembolic disease risk is increased it accounts for few deaths; it is unclear whether this is due to prophylactic treatment with subcutaneous heparin, or that the risk has been exaggerated.

\section{Other issues relating to DKA and HONK}

Shocked patients require catheterisation and measurement of hourly urinary output, but is not necessary in the great majority of patients. Nevertheless, it should be considered if patients have not passed urine after two hours, even if not shocked.

Patients' conscious level may be reduced. If they do not respond to commands, nasogastric tube insertion and aspiration should be considered.

The white cell count may be markedly raised from DKA alone, therefore only give antibiotics if there is clinical evidence of infection.

Silent acute myocardial infarction is not uncommon and an electrocardiogram (ECG) is mandatory in those over 30 years of age with DKA and HONK. However, in severe DKA the ECG may be abnormal and can be mistakenly interpreted as indicating a myocardial infarction. Repeat ECGs may be required and note that serum troponin levels may be mildly raised in DKA, as in many severe illnesses, thus these should be interpreted with care; if necessary cardiology input may be requested.

Pregnant women with DKA are especially worrying as fetal death can result, if over 24 weeks management on the labour ward is essential.

Generalised abdominal pain is not uncommon, but beware the possibility of a surgical underlying diagnosis such as appendicitis and if there are no bowel sounds, or there is vomiting in a moribund patient, nasogastric tube insertion is considered mandatory.

\section{Lactic acidosis}

This is a feared complication and is associated with a high mortality rate. There are two types: type A and type B. The former is not diabetes specific but is commoner in diabetic subjects. It is caused by tissue hypoxia leading to lactate production; diabetic subjects are predisposed to hypoxic conditions such as cardiogenic shock and sepsis. Type B lactic acidosis (also termed aerobic lactic acidosis) is much rarer and is related to inborn errors of metabolism, drugs, and some toxins. Biguanides, such as metformin (presently the only licensed biguanide) do generate a little lactate, but in conditions such as renal impairment it can accumulate and lactic acidosis supervene. The main point to note is that metformin should be avoided in those with "serious" renal impairment; the exact level of serum creatinine used to define avoidance of metformin is controversial and is presently set at about $125-130 \mu \mathrm{mol} / \mathrm{l}$ depending on which guidance one is following. Nevertheless, following the United Kingdom Prospective Diabetes Study study, which showed overall benefit in type 2 diabetes, many practitioners prescribe above these levels, but use a reduced dose.

Patients with lactic acidosis are acidotic and investigation shows low levels of ketonuria/aemia; serum lactate can be measured and is grossly raised in true cases. It should also be noted that serious DKA and HONK cases can have an element of type A lactic acidosis.

\section{Hypoglycaemia}

In the context of diabetes this is due to excess insulin in the absence of enough carbohydrate. It is most common in insulin treated patients, although it may also be caused by sulphonylureas. Recent observational data from the DARTS database in Tayside, Scotland, suggests that hypoglycaemia in type 2 patients is as common as in type 1 and if due to sulphonylureas may be severe and longer lasting than episodes secondary to insulin.

The hierarchy of symptoms and signs starts with very subtle behavioural changes that are not usually detectable by the individual, but may be apparent to others. Next, as the glucose level falls, there are adrenergic symptoms because of epinephrine release (sweating, nervousness, shaking) and then more neuroglycopenic symptoms such as confusion and drowsiness. See box 4 for treatment and box 5 for important notes.

\section{Treatment of high blood glucose in acute coronary syndromes}

The DIGAMI study has demonstrated that intervention to lower a raised glucose level in the context of a proven acute myocardial infarction is important, ${ }^{7}$ particularly in those not previously known to have diabetes. Indeed in these patients the number needed to treat is less than that for thrombolysis in the large intervention studies from the 1980s. This also implies that glucose and insulin infusions may be an important treatment option in other acute coronary syndromes and outcome studies are awaited of treatment of these conditions. It should, however, be remembered that even in post-surgical cases that survival is improved in intensive care units treating raised, but non-diabetic, blood glucose levels. ${ }^{8}$ The authors counsel that all raised blood glucose levels in acute cardiac illness be treated aggressively as diabetic patients now account for about $30 \%$ of coronary care unit admissions. ${ }^{9}$

\section{ADRENAL \\ Acute adrenocortical insufficiency ${ }^{10}$ \\ Causes}

Adrenal insufficiency is surprisingly well tolerated unless additional physiological stress, such as infection or trauma, coexists. Acute adrenocortical insufficiency may be due to primary failure of adrenal function (primary adrenal insufficiency, PAI) or secondary due to lack of adrenocorticotrophic hormone $(\mathrm{ACTH})$ drive from the pituitary (secondary adrenal insufficiency, SAI). Patients with known adrenal failure, or those who have been on long term glucocorticoid treatment are especially at risk (see box 6).

\section{Clinical features}

Clinical features often include weight loss, lethargy, tiredness, weakness, vague abdominal pain, nausea, and vomiting. 
Box 6: Precipitating factors and aetiology of acute adrenocortical insufficiency

\section{General precipitating factors:}

- Intercurrent pyrexial illness.

- Trauma.

- Abrupt cessation of glucocorticoid therapy.

Adrenal specific causes:

- Immune adrenalitis.

- Infectious adrenalitis.

- Tuberculosis.

- Disseminated fungal infection.

- AIDS.

- Haemorrhage:

- Anticoagulant therapy.

- Waterhouse-Friderichsen syndrome.

- Metastases.

- Bilateral adrenalectomy.

- Infiltration:

- Carcinoma/lymphoma.

- Sarcoidosis.

- Amyloidosis.

- Drugs:

- Metyrapone.

- Ketoconazole.

- Etomidate.

Pituitary/hypothalamic causes:

- Tumour.

- Apoplexy.

- Granulomatous disease.

Inherited causes:

- Adrenoleucodystrophy.

- Congenital adrenal hyperplasia.

- Familial glucocorticoid deficiency.

Careful search should be made for precipitating features (box 6). Signs range from mild hypotension with postural drop, to complete cardiovascular collapse and dehydration. Since there is loss of mineralocorticoid secretion in PAI, while this is preserved in SAI, cardiovascular collapse is usually of greater severity in PAI. Untreated PAI is associated with raised plasma ACTH, which causes pigmentation in the buccal mucosa, palm creases, elbows and knees, while in SAI ACTH levels will be normal or low, and pigmentation is not a feature. Electrolyte investigations are consistent with glucocorticoid deficiency and appropriate vasopressin secretion, with hyponatraemia, hyperkalaemia, an increased creatinine, and low plasma glucose. In children hypoglycaemia may be the presenting feature. In undiagnosed and untreated adrenal failure there may be a normochromic, normocytic anaemia, and a raised eosinophil count.

Treatment

If PAI or SAI is suspected blood should be drawn for serum cortisol and plasma ACTH (needs immediate cold centrifugation and freezing at $-20^{\circ} \mathrm{C}$, but arrangements for this must
Box 7: Risk factors for and precipitants of

phaeochromocytoma crisis

\section{Risk factors}

Spontaneous

Associated conditions:

- Multiple endocrine neoplasia type 2.

- Neurofibromatosis type 1.

- Von Hippel-Lindau syndrome.

- Ataxia telangiectasia.

- Tuberose sclerosis.

- Sturge-Weber syndrome.

Precipitating factors

- Spontaneous.

Haemorrhage into phaeochromocytoma

- Other.

Exercise.

Pressure on abdomen.

Urination.

Drugs: guanethidine, glucagons, naloxone, metoclopramide, ACTH, cytotoxics, tricyclic antidepressants.

not delay treatment) and then hydrocortisone is given as $100 \mathrm{mg}$ intramuscularly six hourly, or an infusion of $4 \mathrm{mg} /$ hour intravenously. At these doses mineralocorticoid treatment is not required. Volume expansion with intravenous fluid resuscitation is usually needed, and one litre of normal saline over four hours is a good general starting point, with further replacement as indicated clinically. Any precipitating event should be treated, and broad spectrum antibiotics may be indicated. Hydrocortisone should be continued at this dose for the first 24 hours or until the patient is eating and drinking. At that stage the dose can be reduced to $20 \mathrm{mg}$ orally three times a day, and then to $10 \mathrm{mg}$ three times a day after a further 24-48 hours. Once completely well the usual dose of hydrocortisone, in most patients, is $10 \mathrm{mg}$ on waking, $5 \mathrm{mg}$ at $1200 \mathrm{~h}, 5 \mathrm{mg}$ at $1800 \mathrm{~h}$, and in PAI mineralocorticoid replacement is added (fludrocortisone $0.1-0.2 \mathrm{mg} /$ day).

\section{Further assessment}

Further assessment will depend on the results of the cortisol and ACTH sample obtained in the acute setting. A serum cortisol $<200 \mathrm{nmol} / \mathrm{l}$ is inappropriately low and indicates adrenal insufficiency; serum cortisol $>550 \mathrm{nmol} / \mathrm{l}$ excludes adrenal insufficiency; serum cortisol $>200 \mathrm{nmol} / \mathrm{l}$ $<550 \mathrm{nmol} / \mathrm{l}$ requires further investigation with Synacthen (tetracosactrin) testing. If the plasma ACTH is raised in the context of a low serum cortisol PAI is likely, while a normal or low plasma ACTH suggests SAI. Discrimination between these may be made by a depot Synacthen test and insulin tolerance test. Following the acute event careful patient education is required regarding glucocorticoid therapy, including instructions to double oral hydrocortisone doses at times in intercurrent illness, to carry a steroid card or "Medicalert" bracelet, and the use of a hydrocortisone emergency injection pack (hydrocortisone $100 \mathrm{mg}$ intramuscularly) at home.

In autoimmune PAI coexistent pernicious anaemia may result in macrocytosis. Similarly, although autoimmume PAI may be associated with primary hypothyroidism, a raised thyroid stimulating hormone and low free thyroxine and free tri-iodothyronine are features of glucocorticoid deficiency 
and do not necessarily imply thyroid dysfunction. In this circumstance the thyroid tests will improve following glucocorticoid replacement.

\section{Phaeochromocytoma crisis ${ }^{11}$ \\ Causes}

Associated conditions and factors that precipitate a phaeochromocytoma crisis are given below. Crisis is caused by the action of unopposed high circulating levels of catecholamines acting at adrenoreceptors: $\alpha$-receptors cause a pressor response with increases in blood pressure, while $\beta$-receptor activation has positive inotropic and chronotropic effects. Release of catecholamines from phaeochromocytoma may be episodic. Thus, any patient presenting with acute hypertension and tachycardia should be considered at risk of phaeochromocytoma, especially if young or in an at risk group (box 7).

\section{Clinical features}

Patients will often present with a history of poorly controlled or accelerated hypertension. Presenting features include: hypertension, palpitations, sweating, pallor, pounding headache, anxiety and tremulousness, pulmonary oedema, feeling of impending death, hyperhydrosis, nausea and vomiting, abdominal pain (tumour haemorrhage), paralytic ileus, hyperglycaemia, altered consciousness (hypertensive encephalopathy), myocardial infarction, and stroke. Flushing is not a feature of phaeochromocytoma. The attacks build over a few minutes and then fade more gradually usually lasting for a short time (15 minutes) or being more sustained (60 minutes). Signs of end organ hypertensive damage may be present including: hypertensive retinopathy and papilloedema, left ventricular hypertrophy, renal impairment, and proteinuria. Differential diagnosis includes menopausal vasomotor symptoms, alcohol withdrawal, caffeine intoxication, and thyrotoxicosis. Careful inquiry of precipitating events (box 7) may help in establishing the diagnosis. Examination should include search for associated conditions such as neurofibroma and café au lait spots (neurofibromatosis type 1), and retinal haemangioma (von Hippel-Lindau syndrome).

\section{Biochemical diagnosis}

Biochemical diagnosis is made by collecting urine into acidified bottles for estimation of 24 hour free catecholamines and metanephrines. Plasma metanephrines and catecholamines are also increasingly be used for this diagnosis.

\section{Treatment}

Therapy should not wait for biochemical confirmation. Ideally patients should be monitored on an intensive care unit or the HDU. For treatment of the hypertensive crisis the non-competitive $\alpha_{1} / \alpha_{2}$ antagonist phenoxybenzamine is the drug of choice, with a starting dose of $10 \mathrm{mg}$ orally three times a day, increasing to a maximum dose of $240 \mathrm{mg} /$ 24 hour. Doses above $40 \mathrm{mg}$ three times a day are seldom required. Dose titration is performed every 48 hours until control of blood pressure is achieved. At this stage postural hypotension and tachycardia is almost always present. After the first 48 hours addition of propanolol $40 \mathrm{mg}$ orally three times a day may be added.

Important pharmacological issues in treatment It is vital that 48 hours of $\alpha$-blockade precede $\beta$-blockade to avoid exacerbating a crisis through the unopposed action of catecholamines at $\alpha$-receptors. Side effects to be expected include postural hypotension, nasal stuffiness, drowsiness, and dilutional anaemia. Longer acting competitive
Box 8: Causes of hypercalcaemia

Endocrine:

- Hyperparathyroidism (adenoma, hyperplasia, carcinoma).

- Multiple endocrine neoplasia.

- PTH related protein production by solid tumours.

Neoplastic:

- Carcinoma and bone invasion.

- Myeloma.

Granulomatous:

- Sarcoidosis.

- Tuberculosis.

- Berylliosis.

latrogenic:

- Vitamin D toxicity.

- Thiazides.

- Vitamin A.

Renal failure:

- Tertiary hyperparathyroidism.

- Aluminium toxicity.

Miscellaneous:

- Paget's disease of bone.

- Familial hypocalciuric hypercalcaemia.

- Hypophosphataemia.

$\alpha$-antagonists such as doxazosin, and calcium channel antagonists, while increasingly used for maintenance therapy before operation for phaeocromocytoma, are not recommended for management of crisis. The use of labetalol is not recommended as this has relatively greater $\beta$-blocking action compared to its $\alpha$-blocking action, and hence can even precipitate or worsen phaeochromocytoma crisis.

\section{ACUTE HYPERCALCAEMIA ${ }^{12}$}

Causes of acute hypercalcaemia are laid out in box 8. Severe hypercalcaemia (corrected serum calcium $>3.0 \mathrm{mmol} / \mathrm{l}$ ) requires urgent treatment: in a hospital setting in the UK, $90 \%$ of these are due to malignancy

\section{Clinical features and investigation}

There is often a history of polyuria and polydipsia, and there can be dehydration, bone pain, confusion, anorexia, and constipation. Relevant drug and family histories must be taken. Particular attention must be given to breast examination in females. The cornerstone of differential diagnosis is the measurement of serum parathyroid hormone (PTH). At presentation a sample for PTH should be obtained and stored for future testing. A raised PTH suggests primary hyperparathyroidism and normal or low PTH (in the presence of a grossly increased calcium) malignancy or other cause. Other investigations at presentation should include serum total protein with electrophoresis of immunoglobulins (for myeloma), albumin, phosphate, magnesium, erythrocyte sedimentation rate, full blood count, ECG, and chest radiography. The measurement of vitamin D metabolites is rarely helpful. Further investigations such as serum angiotensin converting enzyme may be considered in difficult cases, with samples 
being obtained ideally before any therapy with glucocorticoids.

\section{Treatment}

Any precipitating drugs should be stopped. The mainstay of treatment is adequate volume repletion with intravenous normal saline, since renal sodium handling is coupled to calcium excretion. Four litres in the first 24 hours are usually needed. Loop diuretics such as furosemide (frusemide), which has calciuretic effects, should only be used after initial volume expansion. Intravenous bisphosphonates, such as sodium pamidronate at a dose of 30-90 mg are extremely effective in the treatment of hypercalcaemia of malignancy, with a duration of action that lasts days to weeks. It is important to remember that serum PTH will rise after an acute fall in serum calcium induced by such treatment and hence it is vital that the initial sample for PTH is obtained before bisphosphonate treatment. Cases of primary hyperparathyroidism should be referred to endocrine surgeons. If granulomatous disease such as sarcoidosis, or vitamin A or D intoxication are considered, corticosteroids (prednisolone 30$60 \mathrm{mg}$ each day) are the drugs of choice.

\section{THYROID \\ Thyroid storm ${ }^{13}$ \\ Causes}

Precipitating factors are listed in box 9. These may be summarised as either being intercurrent illness or direct injury to the thyroid, in a patient with existing thyrotoxicosis. Circulating thyroid hormone levels are generally no higher than in patients with uncomplicated thyrotoxicosis, and free tri-iodothyronine levels may even be normal in individuals who have been ill for several days due to impaired deiodination of thyroxine.

\section{Clinical features}

True thyroid storm is now very rare. There is usually a history of thyrotoxic symptoms and signs over several months, with poor or absent therapeutic control. Cardinal features include: severe tachycardia, fever (usually $>38.5^{\circ} \mathrm{C}$ ), gastrointestinal dysfunction (vomiting, diarrhoea, and occasional jaundice), agitation, confusion, delirium, or coma. Congestive heart failure may occur, particularly in the elderly, and most patients have systolic hypertension. Biochemical features include hyperglycaemia, leucocytosis, mild hypercalcaemia, and abnormal liver function tests. Adrenal reserve may be impaired.

\section{Box 9: Precipitating factors in thyroid storm}

General:

- Infection.

- Non-thyroidal trauma or surgery.

- Psychosis.

- Parturition.

- Myocardial infarction or other acute medical problems.

Thyroid specific:

- Radioiodine.

- High doses of iodine-containing compounds (for example, radiographic contrast media).

- Discontinuation of antithyroid drug treatment.

- Thyroid injury (palpation, infarction of an adenoma).

- New institution of amiodarone therapy.
Treatment

Therapy consists of rapid inhibition of thyroid hormone synthesis and release, inhibition of the peripheral effects of thyroid hormone excess using $\beta$-blockers, treatment of any precipitating illness, and institution of supportive measures. Even with treatment mortality rates of $10 \%-75 \%$ have been reported.

Propylthiouracil, which inhibits thyroid hormone synthesis and conversion of thyroxine to tri-iodothyronine is given by mouth, nasogastric tube, or rectally at a rate of $250 \mathrm{mg}$ every 4-6 hours. Carbimazole is less useful since it does not inhibit conversion of thyroxine to tri-iodothyronine. One hour after starting propylthiouracil, iodide (for example, eight drops of Lugol's iodine every six hours) is given to inhibit thyroid hormone release. This delay allows the antithyroid drug to inhibit thyroid hormone synthesis, which otherwise may be enhanced by unopposed iodide. Alternatively, if available, the radiographic contrast media, sodium ipodate or iopanoic acid, can be given orally, with a loading dose of $2 \mathrm{~g}$ followed by $1 \mathrm{~g}$ daily. These agents also inhibit conversion of thyroxine to tri-iodothyronine.

High doses of $\beta$-blocker should be given, and propranolol at a dose of 80-120 mg every six hours is recommended. High output thyrotoxic cardiac failure will respond to control of the heart rate and therefore $\beta$-blockade should be used (with caution), while in general the response to digoxin and diuretics is poor. Cholestyramine, $4 \mathrm{~g}$ every 6-8 hours, binds thyroid hormone in the gut and thus interrupts the modest enterohepatic circulation of thyroid hormone; its use will lead to a more rapid lowering of circulating thyroid hormones. In exceptional cases, peritoneal dialysis or plasmaphoresis may be needed.

Treatment of any underlying illness follows the usual lines. Supportive treatment includes the use of external cooling, possibly supplemented by chlorpromazine, cautious intravenous fluids, or oxygen as determined by appropriate assessment, and empirical administration of intravenous hydrocortisone $100 \mathrm{mg}$ every eight hours.

\section{Myxoedema coma ${ }^{14}$ \\ Causes}

Precipitating or exacerbating factors for myxoedema coma are listed in box 10. It usually occurs in either previously untreated elderly patients, or those on inadequate or discontinued treatment (the mean age of patients is around 75 years).

\section{Clinical features}

The three main features are: altered mental state ranging from poor cognitive function through psychosis to coma; hypothermia (as low as $23^{\circ} \mathrm{C}$ ) or absence of fever in spite of severe infection (prognosis worsens as the core temperature fall); the presence of a precipitating event.

Box 10: Precipitating factors in myxoedema coma

- Hypothermia.

- Infections especially pneumonia.

- Myocardial infarction or congestive heart failure.

- Cerebrovascular accident.

- Respiratory depression due to drugs (for example, anaesthetics, sedatives, tranquillisers).

- Trauma or gastrointestinal blood loss. 
Other features are present in most patients. The physical signs of hypothyroidism are usually obvious and most patients have respiratory depression secondary to a decreased hypoxic ventilatory drive and an impaired response to hypercapnia: the more severe the latter, the more likely coma is. Cardiac enlargement, bradycardia, decreased ventricular contractility, hypotension, and ECG changes (low voltage, non-specific ST wave changes and sometimes torsades de pointes with a long QT interval) are common. Many patients have anorexia, abdominal pain and distention, and constipation and these changes may rarely lead to paralytic ileus and megacolon.

Biochemical abnormalities include hyponatraemia, normal or increased urine sodium excretion, raised creatine phosphokinase and lactate dehydrogenase, hypoglycaemia, and normocytic or macrocytic anaemia. Thyroid stimulating hormone values may only be modestly raised (and will be normal or low in secondary hypothyroidism) but free thyroxine levels are usually very low.

\section{Treatment}

Even with prompt recognition and treatment, mortality is $20 \%-30 \%$. The three principles of management are rapid institution of thyroid hormone replacement, treatment of the precipitating cause, and provision of ventilatory and other support. There is no agreement on the best way to provide thyroid hormone treatment and direct comparisons between methods are unlikely to be made, given the rarity of the condition. While there is agreement on the need for rapid replacement of thyroid hormones there is no agreement on whether it should be "high dose" or "low dose".

"High dose" regimens may be given as follows: if intravenous thyroxine is available, this can be given as a bolus of $300-500 \mu \mathrm{g}$, followed by $50-100 \mu \mathrm{g}$ daily. Oral thyroxine in similar doses can be given (usually by nasogastric tube), but absorption may be impaired. There is a theoretical concern that conversion of thyroxine to triiodothyronine will be impaired due to the effects of any illness on deiodinase activity, and therefore the use of triiodothyronine instead of thyroxine has been advocated, but this also has the potential to cause adverse cardiac effects when given too rapidly. The usual intravenous dose of triiodothyronine is $10-20 \mu \mathrm{g}$ initially, followed by $10 \mu \mathrm{g}$ every four hours for 24 hours, then $10 \mu$ g every six hours. Oral treatment with similar doses is also possible. A further variation is to give $200 \mu \mathrm{g}$ thyroxine with $10 \mu \mathrm{g}$ triiodothyronine initially, and then tri-iodothyronine $10 \mu \mathrm{g}$ every 12 hours and thyroxine $100 \mu \mathrm{g}$ every 24 hours, until the patient resumes normal thyroxine orally.

Advocates of the "low dose" approach would suggest $25 \mu \mathrm{g}$ of thyroxine daily for a week, or $5 \mu \mathrm{g}$ of tri-iodothyronine twice daily with a gradually increasing dose.

The authors recommend ECG monitoring and careful titration of thyroid replacement therapy against any definite ischaemic ECG abnormalities.

Treatment of the underlying precipitant is usually straightforward. Broad spectrum antibiotics should be considered if there is no obvious cause, as the signs of an infection may be obscure, yet this is the underlying cause in up to a third of cases.

All patients should be admitted to intensive care or the HDU: most patients require ventilatory support for 1-2 days. Hypothermia should be treated with space blankets, since active rewarming leads to circulatory collapse. Cautious volume expansion using intravenous saline usually suffices, but hypertonic saline may need to be considered if the serum sodium is very low $(<120 \mathrm{mmol} / \mathrm{l})$ and intravenous glucose may be required for hypoglycaemia. There is often a degree of temporarily impaired adrenal function, and most authorities

\section{Box 11: Risk factors for pituitary apoplexy}

- Spontaneous.

- Anticoagulation-pre-existing, haemodialysis, cardiac surgery.

- Hypertension.

- Raised intracranial pressure.

- Dynamic pituitary testing-insulin tolerance test, thyroid releasing hormone test, gonadotrophin releasing hormone test, corticotrophin releasing hormone test.

- Drugs-oestrogens, bromocriptine, aspirin.

- Pituitary radiotherapy.

advocate routine intravenous administration of $50-100 \mathrm{mg}$ hydrocortisone every eight hours until recovery.

\section{PITUITARY}

\section{Acute pituitary apoplexy ${ }^{15}$}

\section{Cause}

Pituitary apoplexy is caused by haemorrhagic infarction of a pituitary tumour or less commonly the normal pituitary gland and risk factors are given in box 11. This may cause volume expansion with local mass effect. Since the ocular nerves track through the laterally situated cavernous sinus and the optic chiasm is situated above the pituitary, such mass effect may cause neuro-ophthalmic symptoms and signs. Destruction of pituitary secretory capacity may ensue. Acute pituitary apoplexy is uncommon, occurring in $0.6 \%-$ $9.1 \%$ of all surgically treated pituitary tumours, but is potentially life threatening. Prompt recognition is needed to prevent cardiovascular collapse secondary to pituitary-adrenal hypofunction. There is an equal sex incidence.

\section{Clinical features}

These resemble those of subarachnoid haemorrhage, with severe headache, nausea $(80 \%)$, vomiting $(60 \%)$, photophobia $(50 \%)$, and loss of consciousness $(10 \%)$. Neuro-ophthalmic signs include ocular paresis in up to $70 \%$ of cases with IIIrd $(67 \%)$, IVth $(4 \%)$, and Vth $(29 \%)$ nerve palsies, visual field defects, and even blindness. Pre-existing endocrine dysfunction is present in $20 \%-30 \%$. The patient may have circulatory collapse.

\section{Treatment}

If pituitary apoplexy is suspected (or confirmed by urgent magnetic resonance imaging or computed tomography), hydrocortisone $100 \mathrm{mg}$ intramuscularly six hourly, or $4 \mathrm{mg} /$ hour intravenously should be administered without delay and continued until the crisis is over (see above). Before administration bloods should be drawn for cortisol, prolactin, follicle stimulating hormone, luteinising hormone, oestradiol (women), testosterone (men), sex hormone binding globulin, free thyroxine, thyroid stimulating hormone, insulin-like growth factor-1, and preferably ACTH (needs immediate cold centrifugation and freezing at $-20^{\circ} \mathrm{C}$ ), urinary and plasma osmolality. These can then be analysed routinely and will give important information on pre-existing pituitary function. Further endocrine assessment will be needed once the acute presentation is over. While on high doses of hydrocortisone blood glucose should be monitored and treated accordingly. Otherwise standard cardiopulmonary supportive measures are needed. Urgent neurosurgical opinion should be sought. Early neurosurgical intervention is associated with improved neuro-ophthalmic outcome with 
reported improvements in visual acuity and fields of $86 \%$ and $76 \%$ respectively. In contrast, conservative management is associated with an unpredictable neuro-ophthalmic outcome and recovery of pituitary function is uncommon in most cases regardless of surgical intervention.

\section{MULTIPLE CHOICE QUESTIONS (TRUE (T)/FALSE (F); ANSWERS AT END OF REFERENCES)}

1. In diabetic ketoacidosis:

(A) It is rarely caused by manipulation of insulin dose

(B) The physiological response to DKA tends to lower the blood glucose

(C) Ketones are always detectable in the urine

(D) Measurement of blood gases is usually not required

(E) Intravenous bicarbonate is usually helpful

2. Diabetic ketocidosis/HONK:

(A) Low dose insulin replacement is recommended for the majority of patients

(B) Over zealous fluid replacement has been shown to be responsible for cases of cerebral oedema

(C) Bicarbonate replacement can lower potassium levels and precipitate arrhythmias

(D) Patients with HONK are never acidotic

(E) HONK has been shown to occur in paediatric practice

3. In hypoglycaemia:

(A) Parental therapy is essential

(B) Glitazones and metformin do not cause biochemical hypoglycaemia

(C) Short acting sulphonylurea agents may be a cause

(D) Intramuscular glucagon is the agent of choice in sulphonylurea induced coma

(E) Milk is a good source of readily available carbohydrate

4. In myocardial Infarction/acute coronary syndrome:

(A) The admission blood glucose is inversely related to survival

(B) Studies show that control of blood glucose in newly diagnosed diabetic patients is as important as thrombolysis

(C) Strong clinical evidence exists that it is vital to lower glucose in acute coronary syndrome

(D) The prevalence of diabetes is $10 \%$

(E) Hypoglycaemia secondary to diabetic patients not eating is a major problem

5. Myxoedema coma:

(A) May be precipitated by diazepam

(B) Can only be diagnosed in the presence of bradycardia

(C) Occurs in individuals with a mean age of 65 years

(D) May be treated with thyroid releasing hormone

(E) Has a mortality rate of $40 \%$

6. Thyroid storm:

(A) May be diagnosed by finding a suppressed thyroid stimulating hormone level

(B) Is treated by giving stable iodine and then propylthiouracil

(C) May be accompanied by altered consciousness
(D) Is associated with hyperglycaemia

(E) Is treated with carbimazole in preference to propylthiouracil

7. Acute adrenal insufficiency:

(A) Is excluded by finding serum cortisol $>550 \mathrm{nmol} / \mathrm{l}$

(B) Is treated with dexamethasone in preference to hydrocortisone

(C) In untreated cases is associated with a raised basophil count

(D) Requires large quantities of intravenous dextrose volume replacement

(E) Should be suspected in unwell patients on low maintenance doses of prednisolone

8. Pituitary apoplexy:

(A) Is easily clinically distinguishable from subarachnoid haemorrhage

(B) Needs urgent treatment with parenteral phenytoin

(C) May be precipitated by gonadotrophin releasing hormone testing

(D) Is more frequent in adults

(E) Conservative management is indicated if no neuroophthalmic signs are present

9. Phaeochromocytoma crisis:

(A) Is associated with von Hippel-Lindau syndrome

(B) May be precipitated by chlorpromazine

(C) Flushing is not a feature

(D) Should be treated with propanolol and then phenoxybenzamine

(E) Is usually associated with hyperglycaemia

\section{Authors' affiliations}

M W Savage, Diabetes Unit, Royal Hallamshire Hospital, Sheffield, UK P M Mah, A P Weetman, J Newell-Price, Endocrine Unit, Northern General Hospital and Royal Hallamshire Hospital, Division of Clinical Sciences North, Northern General Campus, University of Sheffield, Sheffield, UK

\section{REFERENCES}

1 Thompson CJ, Cummings F, Chalmers J, et al. Abnormal insulin treatment behaviour: a major cause of ketoacidosis in the young adult. Diabet Med 1995; 12:429-32

2 Guerci B, Benichou M, Floriot M, et al. Accuracy of an electrochemical sensor for measuring capillary blood ketones by fingerstick samples during metabolic deterioration after continuous subcutaneous insulin infusion interruption in type 1 diabetic patients. Diabetes Care 2003;26:1137-41.

3 Kitabachi AE, Fisher JN. Insulin therapy of diabetic ketoacidosis: physiological versus pharmacological doses of insulin and their routes of administration. In: Brownlee M, ed. Diabetes mellitus. Chichester: Wiley, 1981:s95-149.

4 Glaser N, Barnett P, McCaslin I, et al. Risk factors for cerebral edema in children with diabetic ketoacidosis. N Engl J Med 2001;344:264-9.

5 Morris LR, Murphy MB, Kitabchi AE. Bicarbonate therapy in severe diabetic ketoacidosis. Ann Intern Med 1986;105:836-40.

6 Pinies JA, Cairo G, Gaztambide S, et al. Course and prognosis of 132 patients with diabetic non ketotic hyperosmolar state. Diabete Metab 1994;20:43-8.

7 Malmberg K. Prospective randomised study of intensive insulin treatment on long term survival after acute myocardial infarction in patients with diabetes mellitus. DIGAMI (Diabetes Mellitus, Insulin Glucose Infusion in Acute Myocardial Infarction) Study Group. BMJ 1997;314:1512-5.

8 van den Berghe $\mathbf{G}$, Wouters $P$, Weekers $F$, et al. Intensive insulin therapy in the critically ill patients. N Engl J Med 2001;345:1359-67.

9 Baynes KCR, Mclntosh C, Feher MD. Prevalence and management of hyperglycaemia in patients given thrombolysis for acute coronary syndromes. Br J Diabetes Vasc Dis 2003;3:346-8.

10 Trainer PJ, Besser GM. Primary adrenocortical failure. In: Grossman AB, ed. Clinical endocrinology. 2nd Ed. Oxford: Blackwall Science Ltd, 1998:474-83.

11 Werbel SS, Ober KP. Phaeochromocytoma. Update on diagnosis, localisation, and management. Med Clin North Am 1995;79:131-53. 
12 Ross JR, Saunders Y, Edmonds PM, et al. Systematic review of role of bisphosphonates on skeletal morbidity in metastatic cancer. BMJ 2003;327:469-73.

13 Tietgens ST, Leinung MC. Thyroid storm. Med Clin North Am 1995;79:169-84.

14 Braverman LE, Utiger RD, eds. Werner and Ingbar's the thyroid. 8th Ed. Philadelphia: Lippincott, Williams and Wilkins, 2000:1081.

15 Randeva HS, Schoebel J, Byrne J, et al. Classical pituitary apoplexy: clinical features, management and outcome. Clin Endocrinol (Oxf) 1999;51:181-8.

\section{ANSWERS}

1. (A) F, (B) F, (C) F, (D) T, (E) F; 2. (A) T, (B) F, (C) T, (D)

F, (E) T; 3. (A) F, (B) T, (C) T, (D) F, (E) F; 4. (A) T, (B) T, (C)

F, (D) F, (E) F; 5. (A) T, (B) F, (C) F, (D) F, (E) F; 6. (A) F,

(B) F, (C) T, (D) T, (E) F; 7. (A) T, (B) F, (C) F, (D) F, (E) T;

8. (A) F, (B) F, (C) T, (D) T, (E) T; 9. (A) T, (B) T, (C) T, (D) F,

(E) $\mathrm{T}$.

FILLER

\section{If I were to compose a job description}

nevitably, it would be a reflection of my priorities. Having spent my career initially in general practice and, latterly, in the secondary healthcare sector, I have been left with an abiding awareness of the devastation wreaked by hypertension, the end result being stroke, heart failure, ischaemic heart disease, atrial fibrillation, and renal failure, singly and in various combinations, including acceleration of the vascular damage attributable to diabetes.

These adverse outcomes are all potentially preventable through early recognition of hypertension, and through rigorous pursuit of optimum target blood pressures. Recognition of high blood pressure entails education of healthcare professionals in correct techniques of measurement of blood pressure and raising awareness among the management elite that clinical governance encompasses informed choice of blood pressure measuring equipment as well as its regular calibration.

The preventive medical dimension entails education of the general public about dietary health hazards posed by overeating and also by the high salt content of some processed foods. Members of the public should also feel empowered, as of right, to have their blood pressures checked as a matter of routine, in primary as well as in secondary care, irrespective of the reason for the medical consultation, and to have hypertension treated to the optimum target blood pressure. Furthermore should that goal be initially unattainable, it should be an inalienable right for each and every patient to be referred to a blood pressure specialist to have his treatment optimised.

In primary as well as in secondary care management of high blood pressure should be underpinned by audit, encompassing calibration of equipment, technique of blood pressure measurement, lifestyle education of patients, attainment of optimum target blood pressures, referral procedures for refractory hypertension, and diagnostic yield of investigative procedures for suspected secondary hypertension.

For all these reasons it is my firm belief that all NHS trusts would benefit from the services of a specialist whose principal responsibility would be to cover the topics I have enumerated.

O M P Jolobe

Retired Geriatrician, Didsbury, Manchester, UK; oscarjolobe@yahoo.co.uk 